\title{
Conversational Smart Products: A Research Opportunity, First Investigation And Definition
}

\section{Abstract}

This paper approaches the topics of Conversational Design and Conversational Interfaces (CUI) from a product design perspective. While this topic is more widespread in $\mathrm{HCl}$ and $\mathrm{UX}$ design community, there is a lack of insight coming from product design. Given that the integration of CUls and assistants into physical products have the potential to impact product design practice, the paper introduces the term Conversational Smart Products (ConvSP). It analyzes the topic of Conversational Design and the construct of "Product Smartness" and presents a framework to define and analyze ConvSP. The research aims to delve into ConvSP characteristics, deepening how the conversation can be embodied in the product, and how the physical attributes could work in synergy with the verbal dimension. Through a case study research conducted on a selection of 30 existing products with CUls, insight is collected on four different topics. On product smartness and personality; on the level of physical embodiment of the conversation; on request/ response design; and on the tangibility of conversational inputs and outputs. A descriptive definition of Conversational Smart Products is given. Broadly, the research aims to investigate what the role of product designers in the development of ConvSP could be.

\section{Keywords}

Conversational Interfaces, Smart Products, Conversational UX, Product Design, $\mathrm{HCl}$

\section{Introduction}

The idea of building machines capable of behaving and dialoguing as human beings has influenced Artificial Intelligence (Al) since its emergence as a field of research. Since the release of the vocal assistant Siri in 2011, Natural Language Interfaces (NLI) and assistants have become a focal point in both academic and industry research. There are several Conversational User Interfaces (CUI) able to mimic human, turn-based conversation and to use natural language in written or spoken form [1]. Their aim is to provide for a more natural and immediate interaction.

CUls have become increasingly widespread and integrated into physical smart products. This will have an impact on the practice of product design, especially for consumer electronics. The topic of conversational design is gaining importance within the Human Computer Interaction $(\mathrm{HCl})$ community [2] and from the point of view of User Experience (UX) Design. In particular, there is a drive to formalize "Conversational UX Design" as a distinct discipline in UX Design [3-4]. There is a current lack of research on this phenomenon from the perspective of Product Design. Therefore, the paper introduces the term "Conversational Smart Products" (ConvSP), and it delves into their characteristics. It discusses the results of an analysis conducted on 30 existing products with conversational interfaces. It offers product design insight and presents a working definition of ConvSP. 
This paper is part of ongoing research aimed at understanding what could be the role of product designers in the development of conversational smart products. It gives particular attention to how products embed CUls and how the physical attributes could work in synergy with the verbal dimension during the interaction.

The structure of the paper is the following. Section 2 describes the meaning of the term Conversational, illustrates the different conversational interfaces, and outlines how Design is approaching the topic. Section 3 deepens smart products and their characteristics. Section 4 presents the research topic of Conversational Smart Products. Section 5 explains the case study research carried out on 30 existing products with CUls. Section 6 discusses the results, and emphasizes four main topics.

\section{Conversational Interfaces and Conversational UX}

"Conversational Al" is a term used in Artificial Intelligence to represent the study of techniques aimed to create software agents that can engage in natural conversational interactions with humans [5]. The term is historically used applied to interfaces to describe systems that display human-like characteristics and support the use of spontaneous natural language in the form of text or voice.

Conversational interfaces include [1]:

Chatbots. Chatbots are computer programs that process a natural-language input of the user in text form and generate a textual response to the user. Their history goes back to the 1966 with ELIZA, the first chatbot developed at MIT by Joseph Weizenbaum. Chatbots can include visual elements and rich interactions [6]. Chatbots are currently being tested for forms of "Conversational commerce", brand engagement, and integrated in messaging platforms [7].

Vocal interfaces (VUI) are based on a similar technology but have the added complexity of dealing with speech inputs and outputs [8].

Virtual Personal Assistants (VPA) - also called personal butlers or Al assistants - are programs integrated in a device, operating system or app, that can take over a multiplicity of tasks for the users. Assistants are "super bots" that facilitate and manage multiple services [6]. They have a personality and a character, like Amazon's Alexa and Apple's Siri.

\section{Embodied Conversational Agents (ECA) are} agents in form of animated characters on screens. Their aim is to simulate face-to-face interaction with humanlike agents that use their bodies in conversation [9].

"Conversational User Interfaces" (CUI) is one of the terms used to identify Natural Language Interfaces. Cathy Pearl, Head of Conversation Design Outreach at Google [8], states that the term Conversational Design should only be used to identify those systems in which interaction can go beyond one turn. This because human conversation is bidirectional and turn-based. During the conversation, each participant can take the initiative, exchange information, and keep memories of the past turns of the interaction. Machines instead are not always able to keep this memory and go beyond simple one-shot requests, therefore simulating a conversation. For the same reason, Porcheron et al. [10] propose the term "request/response" design to describe interaction with VUls.

Hall [11] instead uses the term Conversational with a broader perspective. According to her: "taking a conversational approach to interaction design requires applying the deeper principles of how humans interact with one another". A truly conversational system should manifest conversational qualities at a deeper level, not only as a façade.

This research uses the term Conversational because it does not focus on the linguistic capabilities of the agent but refers to the emerging discipline of Conversational UX Design. User Experience (UX) is an emergent discipline that focuses on encompassing all aspects of the end user interaction with companies, their services and their products. It is a multidisciplinary field that considers the entire user journey. Usability and User Interface (UI) design are merely subsets of this concept [12].

Conversational UX design means being able to create experiences that work like a conversation. 
Moore et al. [3-4] consider it necessary to define it as a distinct discipline in UX Design. This is because human conversation is a complex system that requires a different skill set/background compared to the development of other interfaces. Designers must become conversation experts and draw knowledge from different disciplines that study human conversation such as Discourse Analysis, Interactional Sociolinguistics, Conversational Analysis, Automatic Speech Recognition (ASR) and Natural Language Processing research (NLP).

The constant increase in products' processing capabilities and connectivity facilitates the diffu-sion of smart products with conversational interfaces. For this reason, Conversational UX De-sign should include contributions from a Product Design perspective.

\section{Smart Products and Product Smartness}

Smart products are a product category that represents internet-connected consumer electronics. Other terms such as intelligent products and connected products have been used to describe the same concept. These objects are reshaping industry boundaries and creating entirely new industries, raising a new set of strategic choices and disrupting value chains [13]. Then, what makes a product smart?

Rijsdijk and Hultink [14] define product smartness as a construct consisting of seven dimensions: autonomy, adaptability, reactivity, multifunctionality, ability to cooperate, human-like interaction, and personality. The level of smartness of a product depends on the extent of its capabilities in one or more of these dimensions. However, these functionalities must be obtained through the use of Information Technologies (IT) for the product to be described as "smart". Although a universally accepted definition of "smart product" does not exist, the term identifies objects that share some common technical characteristics. Different authors propose definitions and technical requirements [15-19].

Smart products share three main characteristics. They are cyber-physical, networked, and with computational intelligence.

Cyber Physical [19]. Smart products blend hardware and software. They are physical objects with a digital representation. Interaction is a key aspect, and it can occur through multiple, multimodal interfaces. For example, there could be a direct interaction with the product combined with an external web application.

Networked. Smart products are networked and uniquely identified. Internet connection makes them part of a larger network of things, people and services. They can communicate with users and/or with other objects. Connectivity can take three forms, that can be present at the same time: one-to-one connection, oneto-many, many-to-many [12]. These connected products are part of the so-called "Internet of Things" [20] and are enhanced by a strong service component [21].

With Computational Intelligence. In contrast to the passive nature of most products, smart products display autonomous and proactive behaviors [15-16]. This blurs the boundaries between product design and robotics, and it leads to reconsider the notion of product agency - that is the ability to act and produce effects according to an intention [22-23]. Computational intelligence may be located in the object (edge intelligence) or even completely outside the physical products, e.g. in the cloud [18]. Computational intelligence enables products to be context-aware, personalized, adaptive and anticipatory. These four characteristics derive from the literature on Ambient Intelligence (Aml), a technological vision developed in the late nineties that envisioned environments infused with technology, sensitive and responsive to the presence of people [24]. Smart products were seen as the building blocks of Ambient Intelligence [17].

The four aspects of computational intelligence according to $\mathrm{Aml}$ are briefly summarized here.

Awareness. In products, awareness can occur in several forms. User-Awareness means that the system is able to recognize and memorize users and their preferences. Contextual-awareness refers to the ability to detect what happens in the environment and to infer user intentions according to the situation. Selfawareness means that the product has and generates new information and memories about itself, its use and purpose. Cultural awareness means that the product is socially appropriate. For example, a talking toy that uses a language appropriate to the children's age. 
Reactive, Adaptive, Anticipatory. Reactiveness is the ability to quickly react to events and environments. Adaptability is the ability to use data to respond and adapt behavior over time, even according to user needs and desires. An anticipatory product can proactively anticipate users' plans and intentions [16], and suggest actions.

Thanks to these features, smart products offer opportunities for envisioning new functionalities and interaction. They raise new challenges for their design, such as designing interactions distributed across multiple devices, in which the focus of the user experience is the service and not the product itself [25].

\section{Research Perspective: Conversational Smart Products (ConvSP)}

Smart products with conversational interfaces and virtual assistants are becoming widespread. McTear, Callejas \& Griol [1] explain that the rising interest in conversational interfaces depends on five main factors: (1) the renaissance of $\mathrm{Al}$ as a field and advances in language technologies; (2) the emergence of the semantic web; (3) the improvement in device technologies; (4) increased access to connectivity; (5) the interest of major companies. In addition, platforms and libraries [5] support the phases of design, prototyping, and development of CUls. For example, the design software Adobe XD added in 2018 the possibility to simulate voice interactions in interface prototypes.

CUls are a topic of interest for Human Computer Interaction ( $\mathrm{HCl})$. In 2018, Clark et al. [2] did a systematic review on the status of speech interfaces in $\mathrm{HCl}$. The study found that current research in $\mathrm{HCl}$ converges on nine topics. These include studies on user/ system speech production, comparisons on different modalities of interaction, investigations on people's experiences with personal assistants, and studies aimed at generating design insight. Their research highlights the lack of design related work and research, outside usability testing and theory-based research.

This research addresses the topic of CUls from a product perspective. In particular, it has three main objectives.
O1: it aims to define "Conversational Smart Products" (ConvSP) and their characteristics;

O2: it aims to deepen how conversation can be embodied in products, and how the physical attributes could work in synergy with the verbal dimension during the interaction;

O3: broadly, it wants to investigate what could be the role of product designers in the development of Conversational Smart Products.

Literature from the disciplines of Human-Computer Interaction, Artificial Intelligence, and User Experience Design contributed to investigate the theme of ConvSP. In $\mathrm{HCl}$, it was given relevance to the subject of speech interaction and Tangible Interaction. Tangible Interaction is an umbrella term denoting systems that rely on embodied interaction, tangible manipulation, physical representation of data, and embeddedness in real space [26].

Tangible interaction is a promising field of research for smart connected products. The study of Tangible Interaction applied to the Internet of Things has been called "Internet of Tangible Things" or loTT [27], and is seen as a way to facilitate the control and understanding of loT objects.

Design researchers are already studying the use of sensory language to communicate information and engage users during user-product interaction [28]. One of the research hypotheses of this paper is that nonverbal, tangible elements could work in synergy with the verbal dimension, and help users to overcome some of the limits of voice-interactions. For example, one limit is that voice is not always the most natural interaction option, or the most socially appropriate. Another issue of VUIs is that users may not know or remember what all the tasks that the system can perform are, because those are not visualized on a display. That is why chatbots and virtual $\mathrm{Al}$ assistants rely on menus and visual elements, like buttons and cards, and smart speakers integrate screens.

\section{Analysis Framework and Case Studies Selection}

As conversational products are getting commercially widespread, the first step of this research consisted in a preliminary analysis based on case studies. Exploratory 
case studies are an appropriate method to inquire little known, poorly understood issues, and whenever the research subject is still emerging. The aim of the investigation was to define ConvSP and gain possible insight for further research.

A framework of five main characteristics was outlined to analyze existing products. The framework is the following (see Table 1).

\begin{tabular}{l|l}
\hline $\begin{array}{l}\text { Type of Conversational } \\
\text { Interface (CUI) }\end{array}$ & $\begin{array}{l}\text { Vocal User Interface } \\
(\text { VUI), Chatbot, Embodied } \\
\text { Conversational Agent (ECA), } \\
\text { Virtual Personal Assistant } \\
(\text { VPA })\end{array}$ \\
\hline $\begin{array}{l}\text { Level of physical } \\
\text { embodiment }\end{array}$ & $\begin{array}{l}\text { Remote, Environmental, } \\
\text { Embedded }\end{array}$ \\
\hline Type of conversation & $\begin{array}{l}\text { User or System initiative, } \\
\text { Task-led or Topic-led } \\
\text { conversation. } \\
\text { Conversation goes beyond } \\
1 \text { turn }\end{array}$ \\
\hline $\begin{array}{l}\text { Input and Output } \\
\text { modalities }\end{array}$ & $\begin{array}{l}\text { Text, Speech, Tangible } \\
\text { interaction }\end{array}$ \\
\hline Product smartness & $\begin{array}{l}\text { Autonomy, Reactivity, } \\
\text { Adaptability, Multi- } \\
\text { functionality, Cooperation, } \\
\text { Awareness, Personality }\end{array}$ \\
\hline
\end{tabular}

Table 1. Framework for the analysis and definition of Conversational Smart Products

\section{The Type of Conversational Interface (CUI)} includes VUIs, Chatbots, ECAs and VPAs. This criterion aims to identify which are the most frequent, and to get insight on how Assistants (VPAs) are integrated in products.

\section{Level of Physical Embodiment of the CUI.}

Fishkin's taxonomy [29] was the starting point to determine where and how conversation takes place in the product. Fishkin proposes a frame-work to analyze the tangible interfaces according to their level of physical embodiment. He distinguishes between four levels of embodiment. "Full" embodiment occurs whenever the output device coincides with the input device. "Nearby" embodiment is characterized by the output taking place near the input object.

"Environmental" embodiment consists of the output being around the user and non-graspable, like sound.

Embodiment is "Distant" if the output happens remotely, such as on another screen or in another room. Based on this framework, three different levels of embodiment for conversation were defined. Conversation is "Remote" when the input or output happens elsewhere. It is "Environmental" when it uses sound and speech. It is "Embedded" when the conversation includes aspects of tangible interaction with the product.

Type of Conversation. Conversation was analyzed in respect to three aspects. The first one is initiative. User-initiative means that the user can do one-shot queries and open-ended prompts, while Systeminitiative means that the system directs the dialogue with slot-filling prompts [1]. The second aspect is the purpose of the conversation. A Task-led conversation aims to accom-plish a task, while a Topic-led one aims to discuss and exchange ideas [6]. Finally, there was an attempt to check if the conversation can go beyond a turn of interaction [8].

\section{The input and output modalities of the} interaction of user and system were deepened to to see if they were only textual and verbal, or if they included tangible interaction.

Lastly, the construct of product smartness was applied to assess whether these products possess the characteristics identified in the literature. Selected product smartness criteria integrate Rijsdijk and Hultink's categories and the principles of Ambient Intelligence described in section 3 of this paper. The seven criteria are: Autonomy (independent action and proactiveness), Reactivity (direct-response to environment and events), Adaptability (over time), Multifunctionality, Cooperation with other devices, Awareness (contextual, of users, of self, of cultural principles), and Personality (having a credible character). 


\subsection{Selection Criteria and Data Collection}

The investigation was carried out on a sample of 30 products with conversational interfaces, or compatible with voice assistants, launched on the market in the last 3-4 years. One requirement was that there would have to be sufficient online material to evaluate their interaction through video and reviews. For this reason, concepts and research projects were discarded. The product selection comes from the most recent tech fairs (i.e. CES 2019, 2018), official lists of products with built-in and compatible assistants (i.e. Alexa, Google Assistant, Siri, Bixby), tech blogs and blogs specialized on conversational interfaces (i.e. Chatbots Magazine, Voicebot, Wired, Tech Insider, etc.).

The 30 case studies belonged to different product categories. They included: a TV remote, camera, door lock, earphones, food scale, commercial robot, light bulb, microwave, musical keyboard, printer, smart clock, fridge, smart outlet, shower, smoke alarm, thermostat, vacuum cleaning robot, washing machine, 2 light switches, 2 smart toys, 3 smart displays, 6 smart speakers.

\section{Results and Discussion: Conversational Smart Products Characteristics and Definition}

Products were analyzed according to the framework described in section 5. The alluvial diagram in Fig. 1 summarizes the case study research in numbers, and highlights the relationships between the different aspects of the framework.

Research findings are summarized in four main topics.

On Product Smartness and Personality. The construct of product smartness proved useful to evaluate smart products. All the analyzed products possessed one or more aspects of smartness. Most of the products (18 p.) possessed three to five aspects at the same time, eight products possessed just one or two, while four possessed six or seven.

Smart products are networked, and through connectivity enable different modalities of interaction. Therefore, Cooperation is the most frequent aspect of product smartness ( 28 out of 30 products), followed by Multifunctionality (23 p.) Awareness \& Adaptiveness (18 p.), Autonomy (15 p.), Reactivity (11 p.) and Personality (6 p.).

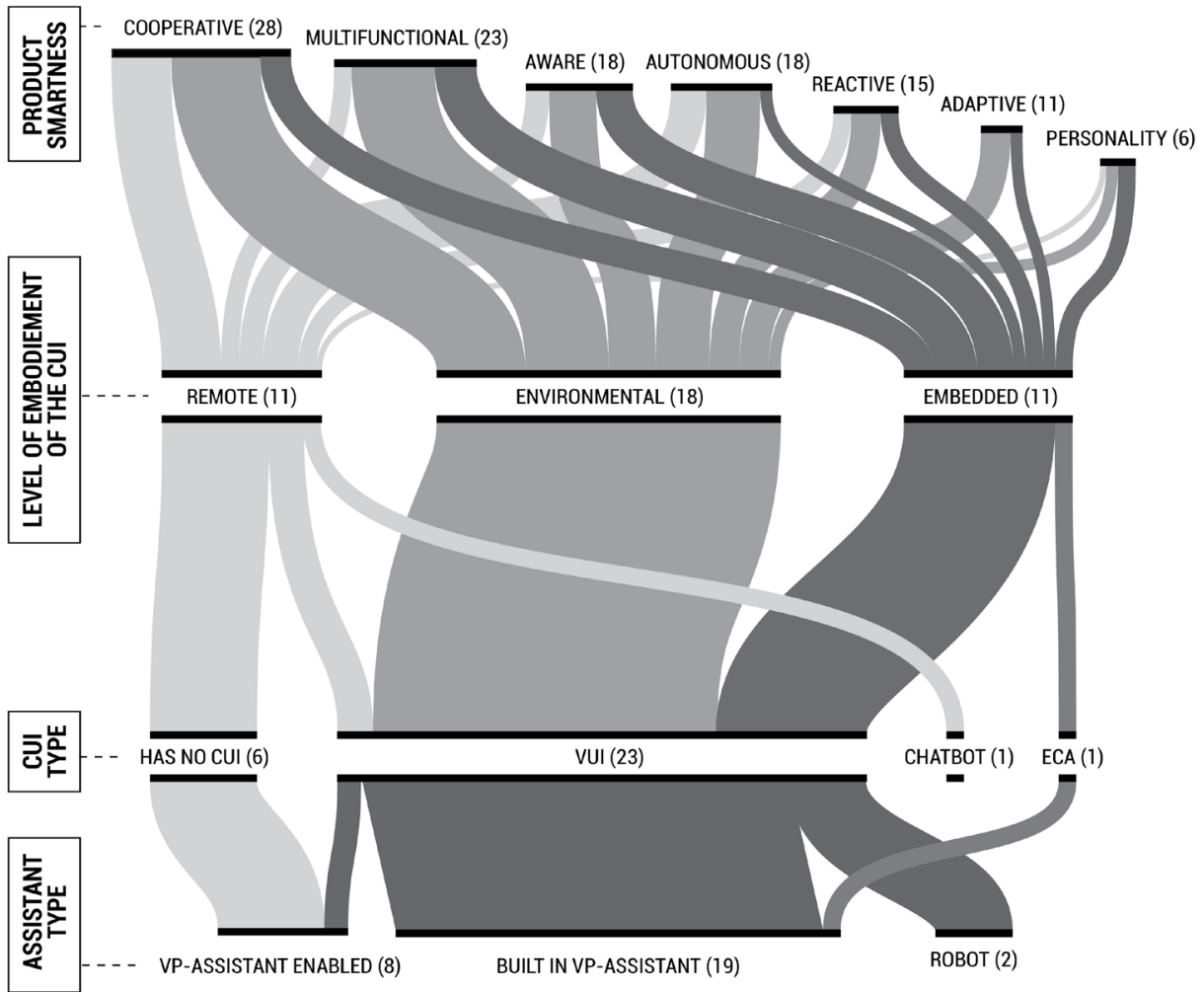

Fig 1. The alluvial diagram summarizes the case study research in numbers, and highlights the relationships between the different aspects of the framework. 
The aspect of personality, while relevant in the design of digital conversational agents and interfaces, it is scarcely present in commercial smart products. It is more frequent for such products to attempt to engage users in topic-led conversations, as done by robots, smart toys, and smart speakers with specific purposes, like teaching languages and playing with children. Most of the examined products integrate existing voice assistants (i.e. Alexa, Google Assistant) and do not create another proprietary character.

\section{Level of Physical Embodiment of the}

Conversation. Conversation happened mainly in an environmental, intangible way (18 products), most frequently through the use of voice inter-faces ( $23 \mathrm{p}$.). In 11 products, conversation took place remotely, according to two types of situations. In the first one, the input or the output of the conversation was given to or from another device. For example, a smart TV remote acted as the speech input, but the output in the form of voice, text, and actions took place on the TV screen. In the second situation, products were simply "VPA assistant-enabled". The products did not integrate CUls. External assistants, in the form of smart speakers of apps, received and processed the commands, while the product only performed actions. Conversation had no impact on the shape of those products. This characterized smart locks, smart outlets, printers, vacuum cleaners, showers, and smart light bulbs. In 11 cases, the products directly integrated the conversation with some tangible elements. Among those, four products used embedded screens to support the conversation. In two cases of robots and smart toys, the conversation was in fact "embodied" by the product through a character.

Voice interfaces were the most frequent CUI (23 p.). Only one product used a chatbot: a washing machine with a chatbot assistant on a dedicated app. Only one smart speaker used an Embedded Conversational Agent: an animated cat, displayed on a screen mounted on top of the device.

Since smart products increasingly embed screens, it is possible to integrate other kinds of CUls beyond VUls. Graphical User Interfaces (GUI) could use the metaphor of conversation in their interactions. This could be useful for those products that guide users in complex procedures.

\section{Conversation Insight: Request/Response Design.}

It was challenging to clearly evaluate the details of the conversation through videos and indirect references. Initiative was left to the user in the majority of samples (29 products), while it was directed in the case of a washing machine controllable via chatbot. Interactions were short and in form of commands. As stated by Porcheron et al. [10], this can be referred to as "request/response design", since there is no real conversation.

Without practical tests, it was not possible to clearly define if the products were able to go beyond the first turn of interaction, as in the definition of Conversational Design given by Pearl [8]. It depends on the capabilities of the conversational agent. The communication capabilities of those products that have built-in assistants will acquire more conversational skills as the assistants are further developed. On the contrary, it is hard to define as Conversational those products are compatible with assistants but do not have CUls themselves. This has been taken into account in the definition of ConvSP.

\section{On Tangibility and Conversational Input \&}

Outputs. The research shows that there are a few aspects of the conversation that are embodied in the product. The first is the "invocation" of the agent, the way in which users can "wake up" the product and start interacting with it. In five products out of 30 , the voice interaction could only be initiated by pressing a physical button. This is also seen as a privacy measure, to ensure that products with VUls are not always "listening". In seven cases, screens were used to support and enrich the conversation. This is particularly true for smart speakers, that are progressively turning into smart displays. This opens up possibilities for multimodal interactions that blend voice interaction and Graphical User Interfaces.

During the conversation, feedback in the form of animated light effects was used to communicate with the user that the agent was listening and producing a response (in eight products). There could be the opportunity to design multimodal feedback that synchronizes to the content of the conversation. For example, the feedback could change in case of greetings, confirmations, and errors. Another opportunity is to design ways to aid users in remembering and visualizing 
the available actions and commands that the product supports.

\subsection{Working Definition of Conversational Smart Products (ConvSP)}

Given the findings of this first investigation into existing products, the working definition of the ConvSP is as follows:

ConvSP are physical, networked products, augmented by a digital counterpart.

They possess one of more aspects of product smartness in the form of Autonomy, Reactivity, Adaptability, Multifunctionality, Cooperation, Awareness, Personality. They embed Conversational User Interfaces (CUI) in the form of Vocal User Interfaces (VUI), Embodied Conversational Agents (ECA), Virtual Personal Assistants (VPA), and chatbots.

There are three levels of physical embodiment of the CUI. It is "Remote" when the input or output happens elsewhere, "Environmental" when sound and speech are used, and "Embedded" when there the conversation includes tangible interaction with the product. A ConvSP must clearly display or suggest through its physical form and feedback, that is conversationally enabled.

\section{Conclusions}

This paper approaches the topic of Conversational Design and Conversational Interfaces (CUI) from a product design perspective. It introduces the term Conversational Smart Products (ConvSP) to describe those physical products that embed conversational interfaces, and offers a descriptive definition.

Broadly, this work is part of a research that aims to investigate what could be the role of product designers in the development of conversational smart products.

The paper proposes a framework for the analysis and definition of Conversational Smart Products which considers the type of CUI, the level of physical embodiment of the conversation, the type of conversation, the input and output modalities, and the overall product smartness. It begins to investigate how the conversation can be embodied in the product, through a case study research conducted on a selection of 30 existing products with CUls. A limit to the present study is that it only investigates smart products using indirect resources such as videos and reviews.

Therefore, the evaluation of the interaction may not be complete or completely correct.

It is a preliminary study that proposes four different topics that can be relevant in the design of ConvSP. The first point is that ConvSP possess one or more aspects of product smartness. The aspect of personality is more relevant for those products that engage in topic-led conversations, such as robots and smart toys. The second point is that interaction with ConvSP is mostly environmental, and happens via VUI. Since smart products embed screens, there is space to integrate other kinds of CUls, such as chatbots and ECAs, and to design Graphical User Interfaces (GUIs) that use the metaphor of the conversation.

The third point is that the ability to converse strictly depends on the conversational agent. Therefore, products with built-in assistants will have the most advanced capabilities. Products that are only "assistantenabled", without any impact on the product, can hardly be considered as conversational.

The fourth and last point is tangibility. There are different elements that show potential for tangibility: the design of the "invocation" of the agent, the design of the system's outputs, and the design of systems to guide users in remembering and visualizing the available actions and commands.

Further research could explore the firsthand experience of designers that are already involved in related projects, with the goal of investigating their role in the development of ConvSP. Other activities could include practical tests with ConvSP, and design activities aimed at exploring how physical attributes could work in synergy with the verbal dimension.

\section{References}

1. McTear, M., Callejas, Z., Griol, D.: The conversational interface: Talking to smart devices. Springer (2016)

2. Clark, L., Doyle, P., Garaialde, D., Gilmartin, E., Schlögl,

S., Edlund, J., ... \& Cowan, B.: The State of Speech in $\mathrm{HCl}$

Trends, Themes and Challenges. arXiv preprint (2018) 
3. Moore, R. J., Arar, R., Ren, G. J., \& Szymanski, M. H.: Conversational UX design. In Proceedings of the $2017 \mathrm{CHI}$ Conference Extended Abstracts on Human Factors in Computing Systems, pp. 492-497. ACM (2017)

4. Moore, R. J., Szymanski, M. H., Arar, R., \& Ren, G. J.: Studies in Conversational UX Design. Springer (2018)

5. Ram, A., Prasad, R., Khatri, C., Venkatesh, A., Gabriel, R., Liu, Q., ... \& King, E.: Conversational Al: The science behind the alexa prize. arXiv preprint (2018)

6. Shevat, A.: Designing bots: Creating conversational experiences. O'Reilly Media, Inc. (2017)

7. Gentsch, P.: Conversational Al: How (Chat) Bots Will Reshape the Digital Experience. In Al in Marketing, Sales and Service, pp. 81-125. Palgrave Macmillan, Cham (2019)

8. Pearl, C.: Designing Voice User Interfaces: Principles of Conversational Experiences. O'Reilly Media, Inc. (2016)

9. Cassell, J., Sullivan, J., Churchill, E., Prevost, S.: Embodied conversational agents. MIT press (2000)

10. Porcheron, M., Fischer, J. E., Reeves, S., Sharples, S.: Voice Interfaces in Everyday Life. In Proceedings of the 2018 CHI Conference on Human Factors in Computing Systems, pp. 640, ACM (2018)

11. Hall, E.: Conversational design. A book apart (2018)

12. Norman Nielsen group, "The Definition of User Experience (UX)". Retrieved from hwww.nngroup.com/articles/ definition-user-experiencel. Accessed 21-05-19

13. Porter, M. E., \& Heppelmann, J. E.: How smart, connected products are transforming competition. Harvard business review, 92(11), pp. 64-88 (2014)

14. Rijsdijk, S. A., \& Hultink, E. J.: How today's consumers perceive tomorrow's smart products. Journal of Product Innovation Management, 26(1), pp. 24-42 (2009)

15. Gutierrez, C., Garbajosa, J., Diaz, J., Yague, A.: Providing a consensus definition for the term "Smart Product." Proceedings of the International Symposium and Workshop on Engineering of Computer Based Systems, pp. 203-211 (2014)

16. Maass, W., \& Janzen, S.: Dynamic Product Interfaces: A Key Element for Ambient Shopping Environments. 20th Bled eConference (2007)

17. Mühlhäuser, M., Ferscha, A., \& Aitenbichler, E., (eds): Constructing Ambient Intelligence: Aml 2007 Workshops Darmstadt, Germany, Revised Papers. Vol. 11. Pp. 156-164. Springer Science \& Business Media (2008)

18. Meyer, G. G., Främling, K., \& Holmström, J.: Intelligent products: A survey. Computers in industry, 60(3), pp.137148 (2009)
19. Abramovici, M.: Smart products. CIRP Encyclopedia of Production Engineering, (2014)

20. Greengard, S.: The internet of things. MIT Press (2015)

21. Brehm, L., \& Klein, B.: Applying the Research on ProductService Systems to Smart and Connected Products. In International Conference on Business Information Systems, pp. 311-319. Springer (2016)

22. Kaptelinin, V., Nardi, B. A.: Acting with technology: Activity theory and interaction design. MIT press (2006)

23. Cila, N., Smit, I., Giaccardi, E., \& Kröse, B.: Products as Agents. Proceedings of the $2017 \mathrm{CHI}$ Conference on Human Factors in Computing Systems - CHI '17, pp. 448-459 (2017)

24. Aarts, A., De Ruyter, B.: New research perspectives on Ambient Intelligence. Journal of Ambient Intelligence and Smart Environments. pp. 5-14 (2009)

25. Rowland, C., Goodman, E., Charlier, M., Lui, A., \& Light, A.: Designing Connected Products: UX for the Consumer Internet of Things. O'Reilly Media, Inc. (2015)

26. Hornecker, E., Buur, J.: Getting a grip on tangible interaction: a framework on physical space and social interaction. In Proceedings of the SIGCHI conference on Human Factors in computing systems, pp. 437-446, ACM (2006)

27. Angelini, L., Lechelt, Z., Hornecker, E., Marshall, P., Liu, C., Brereton, M., ... \& Mugellini, E.: Internet of Tangible Things: Workshop on Tangible Interaction with the Internet of Things. In Extended Abstracts of the $2018 \mathrm{CHI}$ Conference on Human Factors in Computing Systems. ACM (2018)

28. Bergamaschi, S., Rampino, L. R., \& Dijk, J. V.: Designing Dialogs between Users and Products through a Sensory Language. In DeSForM 2017, pp. 149-163 (2017)

29. Fishkin, K.P.: A taxonomy for and analysis of tangible interfaces. Personal and Ubiquitous Computing. 8(5), pp. 347-358 (2004) 